\title{
SMART INTERNET CONNECTED MOBILE PHONE REMOTE FOR MONITORING AND CONTROLLING OF HOUSE AND HOUSEHOLD APPLIANCES
}

\author{
Pandiaraj R, \\ Department of Electrical and Electronics Engineering, \\ St. Peter's Institute of Higher Education and Research, \\ Avadi, Chennai, India \\ pandiaraj.r@gmail.com \\ Dr Rani Hemamalini R, \\ Department of Electronics and Communication Engineering, \\ St. Peter's Institute of Higher Education and Research, \\ Avadi, Chennai, India \\ ranihema69@gmail.com
}

\begin{abstract}
Thinking about the ON/OFF state of electrical appliances, gas regulator and worrying about the safety of costly things in home are common to everyone. Carelessness or failing to off the appliances sometimes may cause minor or major accidents in home. The modern technologies available today will gives lot of solutions to overcome this problem. This paper gives details about a device built with sensors and Internet of Things (IoT), ZigBee wireless technology and a mobile phone application for monitoring and controlling. IoT means, things that are connected to internet and capable of accessing from anywhere. The device monitors the house with the help of sensors connected to it and updates information to the owner's mobile phone through internet connection available in the house. The same way owner can control the home appliances and know its status from the mobile application. This device will reduce the burden of the owners, by keeping them aware of their home anywhere anytime. This device can also be useful for elderly and physically challenged people to control the appliances from mobile phone which is acting as a remote control in this device.
\end{abstract}

Keywords: IOT, ZigBee, Home automation, Security and Control, ESP 8266, Arduino.

\section{INTRODUCTION}

Home automation means automating the various processes in our home by means of technologies. It includes applications such as home security, automated lighting, remote control of appliances, ambient monitoring, baby monitoring and pet feeding etc. This will enrich the daily life of people by making the process easy.

Home automation has evolved in different timings with various technologies such as infrared (IR), power line [1], RF, bluetooth [2-3], ZigBee, internet. In initial stages, infrared (IR) based wireless remote was used to control the electrical loads like air-conditioners [4], television. The drawback of this remote control is, it needs line of sight communication between the transmitter and receiver and the communication distance is very low. In [5-6] the IR based wireless communication is replaced by ZigBee wireless protocol and created a wireless home network to control the electrical appliances present in our home.

Web server based ambient monitoring system was designed in [7] to monitor the parameters such as temperature, humidity, water level and smoke. In 
this internet based device, web page will be loaded in the flash memory of the device and this will act as a web server. From any internet browser the device can be accessible by its internet protocol address. We needed a internet facility available PC for monitoring this ambient situations. Internet supported universal remote controller was designed by author in [8] for home automation. This also has a drawback such as we need to carry a special device for control and monitoring our home devices.

The rapid growth in the development of internet technologies such as IoT and cloud, created a new path for the design of home automation. IoT means various devices, things, objects and sensors that are connected together through communication infrastructure to give value added services [9]. According to a survey from McKinsey Global Institute, IoT is having huge impact on global economic [10]. IoT is applicable in various domains like wearables, healthcare, smart environment, smart home and transportation etc [11].

The home automation system discussed in this study uses the IoT technology. It overcomes the distance related problem. And everyone is having smart mobile phone nowadays. So the system avoids the need of separate remote control device for monitoring and controlling. It gives enough information at right time about an incident, if anything happens at home. The system provides SMS alert to the owners and public service departments like police station and fire service station depends on the nature of incident. For example if the device finds any theft related incident it will alert the house owner and nearby police station. And similarly if it finds any fire related incident, it will alert both owners, fire service station and police

\section{METHODS AND MATERIALS}

The entire design consists of 2 units such as home device and an application running in the owner's mobile phone. Home device unit consists of a Home Gateway Unit (HGU) and different Sensor Units (SU) and Control Units (CU). HGU acts as a middle man between owner and other units present in the home. It receives commands from owner and passes it to the $\mathrm{CU}$ and similarly relays the information received from SU to owner and other public service departments (PSD).

Various sensors available in SU are human motion sensor (HMS), fire sensor, door break sensor (DBS) and gas leak sensor and CU consists of electrical relays to control the appliances. Overall representation of the system is shown in Fig. 1. Detailed block representation of HGU, SU and CU are shown in Fig. 2, Fig. 3 and Fig. 4 respectively.

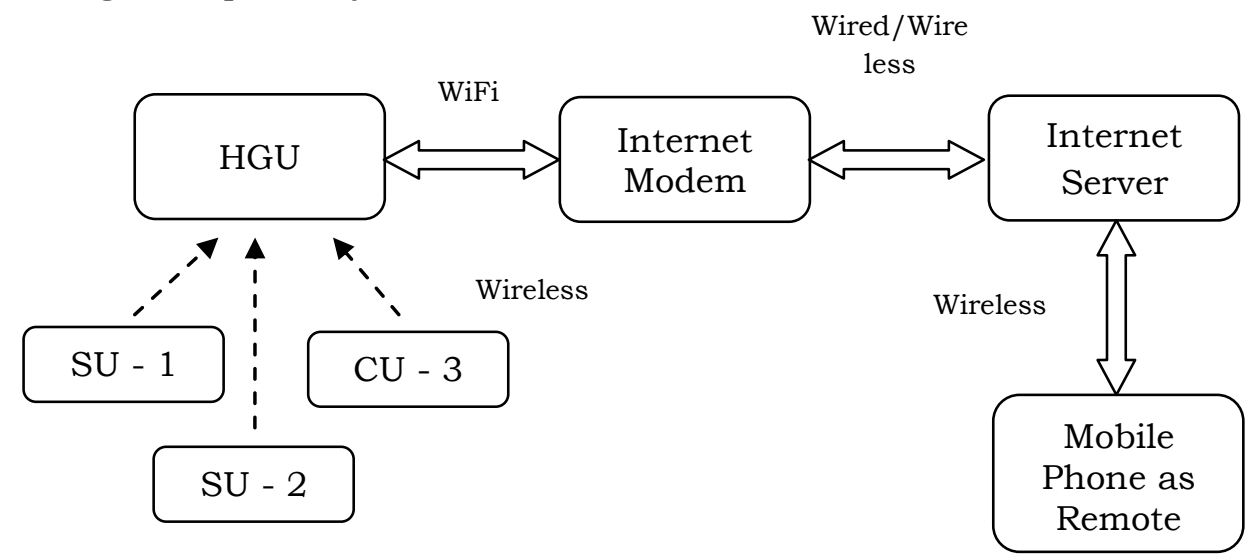

Fig. 1 Overview of the System 


\section{A. HGU}

As shown in Fig. 2, HGU consists of an arduino based microcontroller platform, ZigBee wireless transceiver for enabling wireless communication between other SU and CU. And it consists of a WiFi module (ESP 8266) to send and receive data to the internet through internet modem available in home. The GSM modem available in HGU is used to send mobile SMS alert to owner and PSD. Arduino is an open source microcontroller platform used to create innovative applications easily and in short time [12].

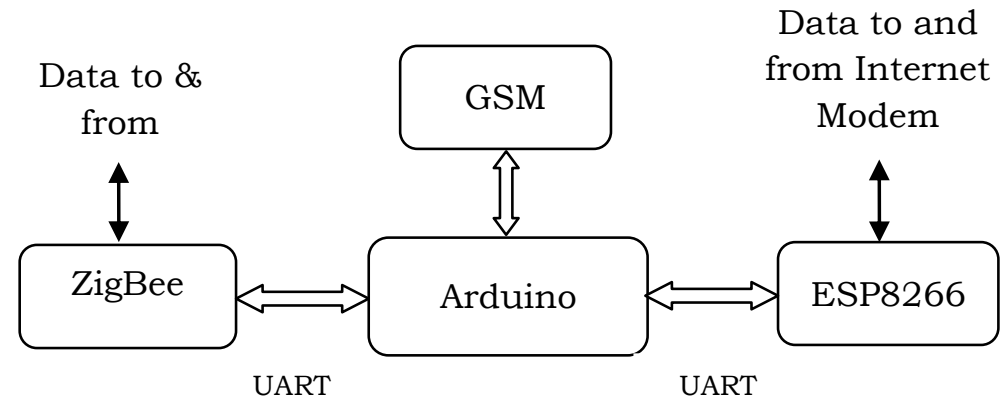

Fig. 2 Block diagram of $\mathrm{HGU}$

\section{B. SU}

Detailed block view of SU is shown in Fig. 3. It consists of an arduino, a ZigBee transceiver to send sensor information to HGU, sensors and a camera trigger to ON the camera module to record the abnormal incidents if found. IR based fire sensor and a smoke sensor (MQ2) are used to find the fire accident. Gas sensor is used to detect the leakage of liquefied petroleum gas. Vibration sensor is used to detect the door breaking event and passive infrared (PIR) sensor is used to detect human motion inside the home. Controller will trigger a siren to alert the neighbors in case of any alerts received from sensors.

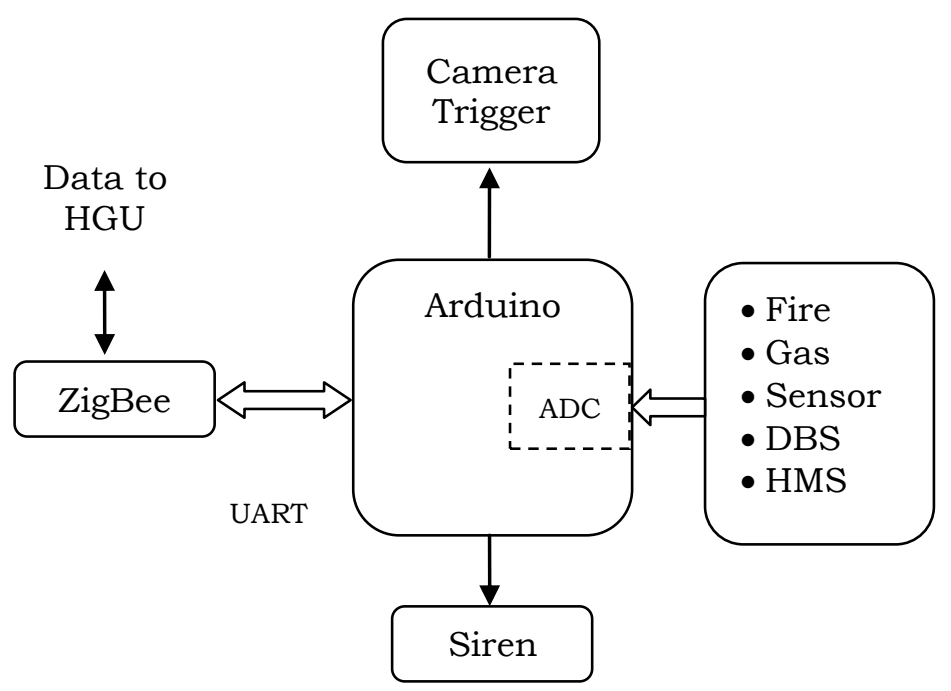

Fig. 3 Block Diagram of SU 


\section{C. $\quad \boldsymbol{C U}$}

It consists of ZigBee, an arduino, relays and its driver as in Fig. 4. The electrical appliances are connected to the corresponding relays. User commands are received from HGU through ZigBee and controller triggers corresponding relay to turn ON/OFF the appliance.

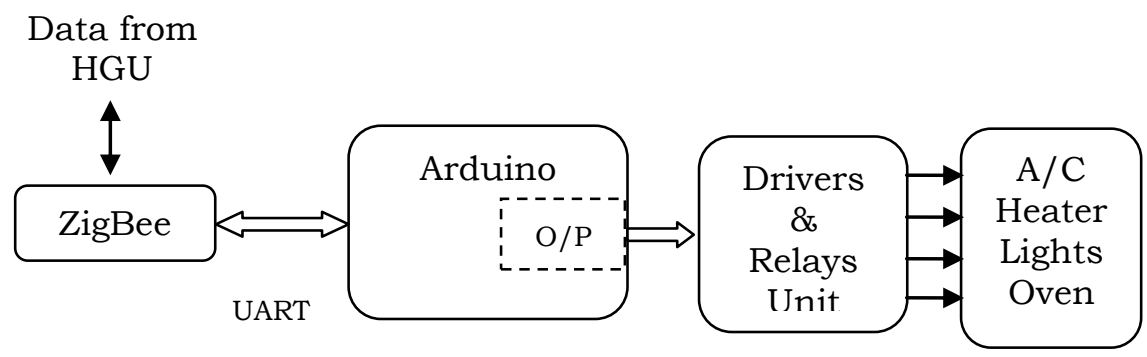

Fig. 4 Block Diagram of $\mathrm{CU}$

\section{IoT Cloud Server and Smart phone Application}

Many cloud platforms are available. In our project Blynk cloud server and its smart phone application are used because it is simple and easy to use. The need of cloud server is to authorize the smartphone application with the hardware device, to establish communication and to manage the data transmission between them. The device can connect to the internet through WiFi or LAN and here ESP8266 WiFi module is used.

The smartphone application is used to create custom user interface (UI) as per the design requirements. It is used to read the data coming from the device and send the user commands to the device. An authentication token will be issued by the server for the device during creation of the project in this application. This token is unique and it is used to connect the hardware to the smartphone application. Blynk library running in the microcontroller will handle the specific communication protocol used in the communication between device and cloud server

\section{RESULTS AND DISCUSSION}

Internet connected and controllable smart device for automating the household appliances is designed and it is shown in Fig 5. The UI designed using the Blynk application for mobiles is shown in Fig 6 . The UI consists of 4 buttons to control 4 electrical appliances and 4 virtual alert LEDs to show the status of sensors. After turn ON the devices the HGU is checking the internet connection and then pairs with owner's Blynk application account with the help of a specific token generated to their mobile during log in session. It avoids the unknown people accessing and controlling our HGU. Once the pairing process is completed, owner can command to the devices through UI designed in their mobile and similarly the HGU can read user commands from and send alerts to UI. Upon receiving abnormal alerts from SU, the HGU triggers the camera to capture and record the videos and it sends alert SMS to the owner and PSD. 


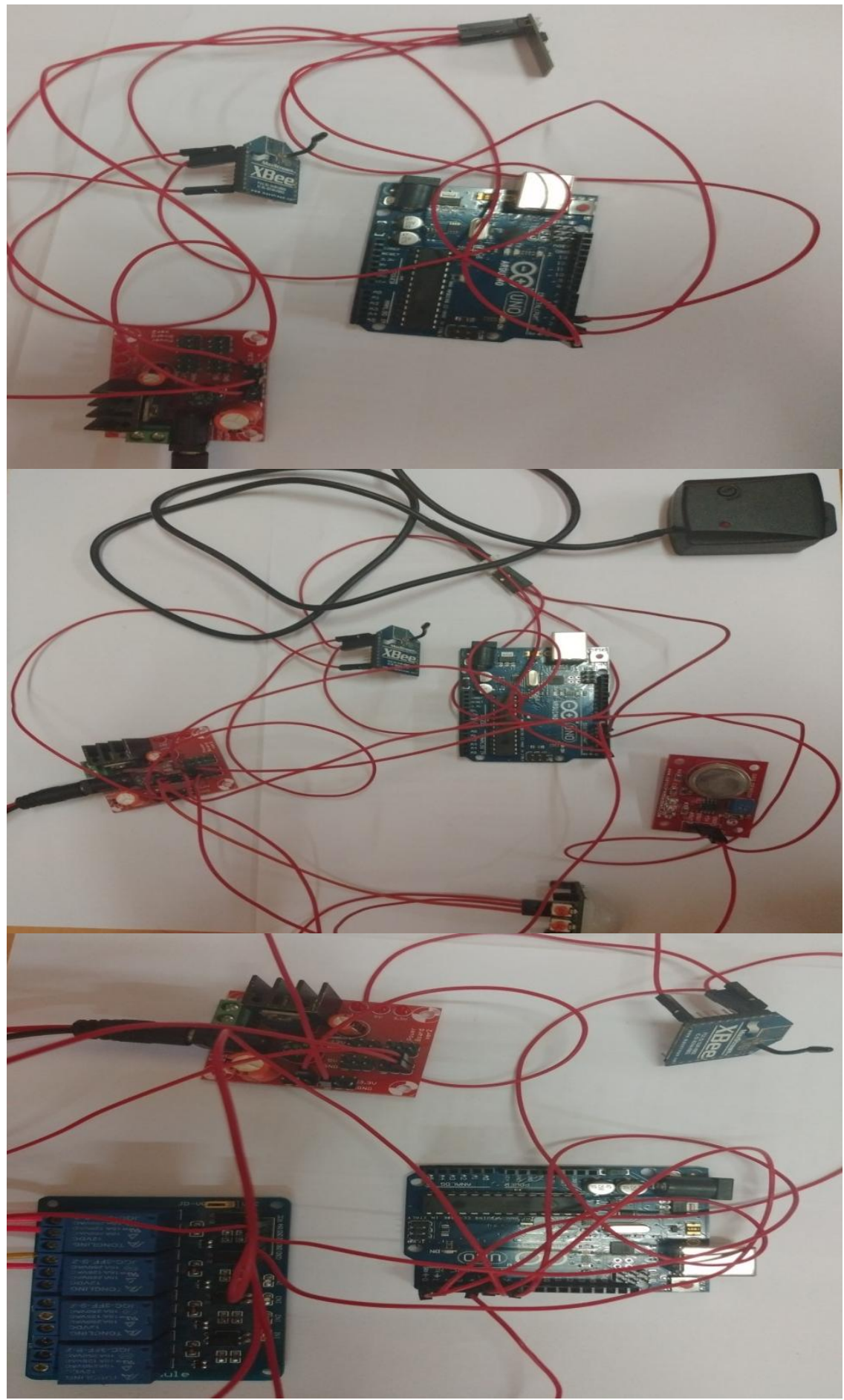

Fig. 5 Photo view of HGU, SU and CU

The button is changing its label to ON/OFF depend on pressing it. The virtual LED nearer to each button is controllable by the HGU and it triggers the corresponding virtual LED to show the status of appliance. The alert LEDs present in 3rd column are used for showing fire alert, motion alert, theft alert and gas leak alert. These LEDs also controllable by HGU and it triggers the LEDs depends on 
sensor status receiving from SU. The virtual LCD at the bottom of UI is used to show text alerts to the owners.

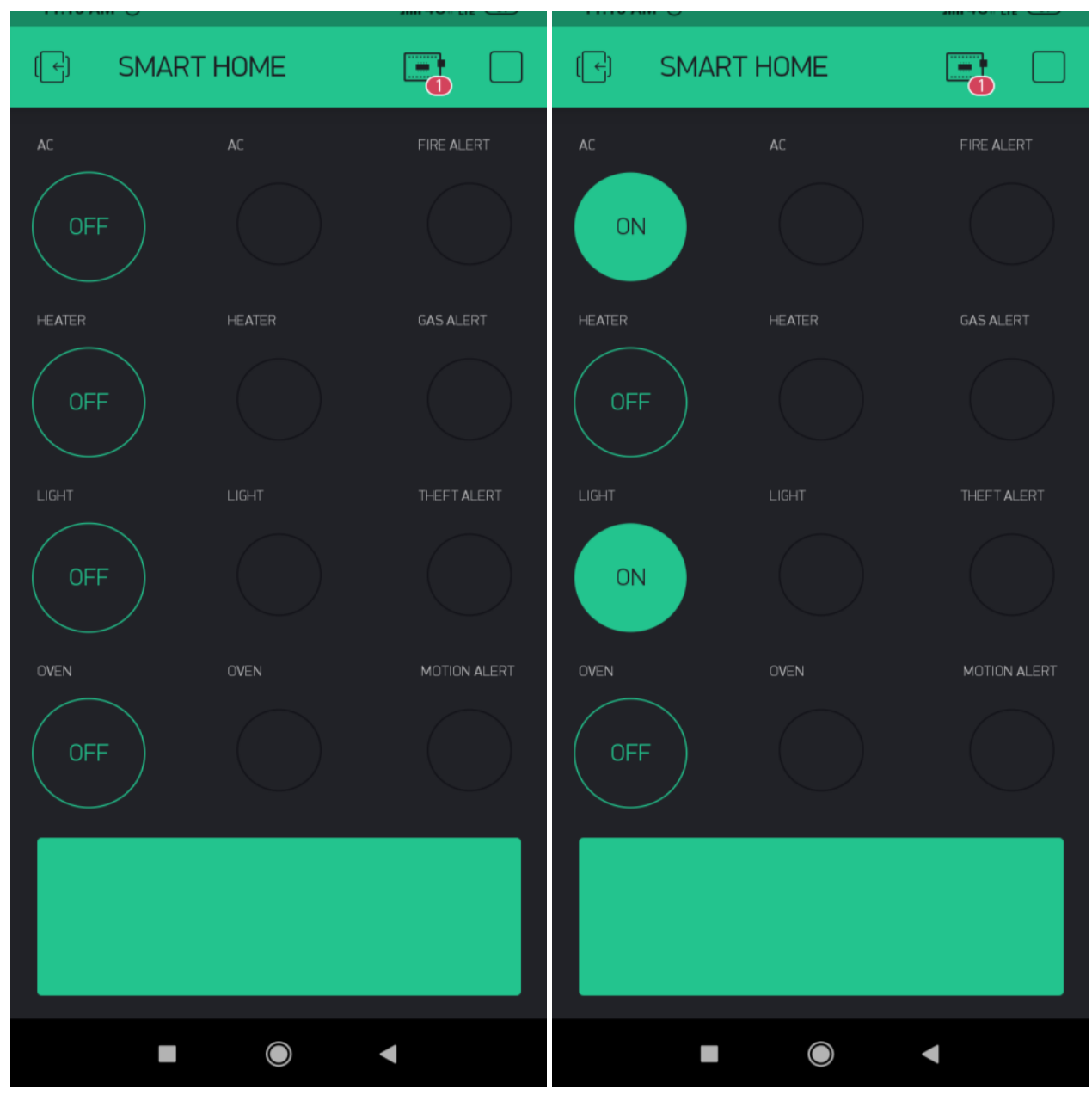

Fig. 6 UI designed using BLYNK application

\section{CONCLUSION}

The device for automating the various requirements of home is designed and tested. All the functionalities of the device are checked through the Blynk mobile application which is connected to the device through internet. The device has successfully controlled the appliances by receiving input from owner. And also it has informed the status of sensors to the owner. The response time is tested for each case and it is found that the device responds in couple of seconds for each input. The videos recording during the time of abnormal cases will be helpful for investigations. The siren helps the neighbors to get alert and act fast. The SMS sending to PSD during the abnormal situation will help them to reach the spot as early as possible and to bring the situation to its normal. 


\section{REFERENCES}

[1]. L.A. Bertsch, "Development tools for home automation", IEEE transactions on consumer electronics, Vol. 36, No. 4, 1990, pp. 854-858.

[2]. R. Shepherd, "Bluetooth wireless technology in the home", Electronics \& Communication Engineering Journal, Vol. 13, No. 5, 2001, pp. 195-203.

[3]. Y. Tajika, T. Saito, K. Teramoto, N. Oosaka, and M. Isshiki, "Networked home appliance system using Bluetooth technology integrating appliance control/monitoring with Internet service", IEEE Transactions on Consumer Electronics, Vol. 49, No. 4, 2003, pp. 1043-1048

[4]. I.G. Park, "The remote control system for the next generation air conditioners", IEEE Transactions on Consumer Electronics, Vol. 47, No. 1, 2001, pp. 168-178.

[5]. I.K. Hwang, D.S. Lee, and J.W. Baek, "Home network configuring scheme for all electric appliances using ZigBee-based integrated remote controller", IEEE Transactions on Consumer Electronics, Vol. 55, No. 3, 2009, pp. 1300-1307.

[6]. K. Gill, S.H. Yang, F. Yao, and X. Lu, "A zigbee-based home automation system", IEEE Transactions on consumer Electronics, Vol. 55, No. 2, 2009, pp. 422-430.

[7]. I. Klimchynski, "Extensible embedded web server architecture for Internetbased data acquisition and control" IEEE Sensors Journal, Vol. 6, No. 3, 2006, pp. 804-811.

[8]. T. Kim, H. Lee, and Y. Chung, "Advanced universal remote controller for home automation and security", IEEE Transactions on Consumer Electronics, Vol. 56, No. 4, 2010, pp. 2537-2542.

[9]. C. Perera, C.H. Liu, and S. Jayawardena, "The emerging internet of things marketplace from an industrial perspective: A survey", IEEE Transactions on Emerging Topics in Computing, 2015, Vol. 3, No. 4, pp. 585-598.

[10]. A. Saint, "Where next for the Internet of internet of things?", Engineering \& Technology, Vol. 10, No. 1, 2015, pp. 72-75.

[11]. A. Kamilaris, and A. Pitsillides, "Mobile phone computing and the internet of things: A survey", IEEE Internet of Things Journal, Vol. 3, No. 6, 2016, pp. 885-898.

[12]. https://www.arduino.cc/en/Guide/HomePage 